\title{
Application of pre-medicated collagen sponges with regenerative biomaterials for management of an infected extraction socket
}

\author{
Mohammed E. Sayed ${ }^{1}$ \\ ${ }^{1}$ Jazan University Faculty of Dentistry
}

March 5, 2022

\begin{abstract}
Localized infection of the extraction socket can compromise bone quality and quantity within the socket and bone support for the adjacent dentition, precluding immediate rehabilitative interventions. The use of local scaffolds containing effective antimicrobial agents may help to control infection for successful regeneration and implant placement.
\end{abstract}

\section{Introduction}

Exodontia is associated with complications such as postoperative infection, accounting for $1.4 \%$ of treated cases. ${ }^{1}$ A large-scale prospective study reported very low incidence of postoperative infection in teeth with preoperative history of infection. ${ }^{2}$ As an alternative to tooth replacement, implant-supported restorations may comprise a viable treatment modality for rehabilitation of partially edentulous patients and have shown higher success rates for single-tooth replacement. ${ }^{3} \mathrm{Chen}$ and Buser ${ }^{4}$ published a classification system for the implant-placement timing following tooth extraction and categorized the placement as immediate, early with soft-tissue healing, early with partial bone healing, or delayed. Although immediate dental-implant placement in fresh extraction sockets is an established treatment modality, ${ }^{5-7}$ a recent meta-analysis ${ }^{8}$ reported that immediate implant placement is associated with a higher risk of early implant loss. The success rate could significantly decrease by the presence of risk factors leading to early implant failure at second-stage surgery, and infection was the most significant risk factor with the highest odds ratio compared to other factors. ${ }^{9}$ Immediate implants can offer predictable results in sites with history of periapical and periodontal infections, as careful debridement is performed before implant insertion. ${ }^{10}$ Moreover, infection of the adjacent teeth may complicate the success of immediate implants in short- and long-term follow-up assessments. ${ }^{11}$ Thus, socket infection may compromise the anatomical structures necessary for primary implant stability and the success of immediate implants. The inter-radicular bone in molar sites, for instance, is a crucial parameter playing a role in dental implant stability and success. ${ }^{12}$ Therefore, in such cases, guided bone regeneration may be the first step to prepare the implant bed for later surgical placement. ${ }^{13}$

In addition to mechanical debridement of the infected socket, pharmacological interventions may be helpful for management of local aseptic conditions. The potential benefits of systemic antibiotic treatment following guided tissue and bone regeneration have been questioned. ${ }^{14-17}$ The application of postoperative systemic antibiotics may be helpful in reducing discomfort following the guided tissue-regeneration procedure. ${ }^{18}$ Local application of chlorhexidine chips ${ }^{19}$ and metronidazole gel ${ }^{20}$ showed promising results in guided tissue regeneration of periodontal defects. Nonetheless, applying these agents for guided bone regeneration of compromised extraction sockets as a preparatory step for future implant therapy requires evaluation. This report aims to introduce the strategic application of pre-medicated collagen sponges containing both chlorhexidine and metronidazole together with a particulate bone graft and collagen membrane for guided tissue and bone regeneration following extraction of a split mandibular first molar with purulent infection, fistula opening, and severe vertical bony defect of the adjacent second premolar. 


\section{Case Description and Results}

A 36-year-old Saudi Arabian woman presented to a private clinic with a chief complaint of tooth mobility and pain. The patient was medically fit with dental history of a few composite restorations performed 2 years previously. No medication usage was reported. Intraoral clinical examination revealed that the intraoral soft and hard tissues were within the normal limits. Tooth \#19 presented with a vertical fracture extending deep below the gingival margin with noticeable mobility of the broken proximal halves. A fistula opening with purulent discharge on pressure palpation was detected on the buccal gingival tissue. A vitality test using the cold-testing technique was performed on tooth \#20 and revealed a vital pulpal response. Radiographic examination revealed that tooth \#19 had a deep fracture line extending halfway between the cementoenamel junction and mesial root apex. A large peri-apical/periodontal infection was observed around the fracture area and distal root surface of tooth \#20 (Fig. 1). Probing of the mesial aspect of the fractured tooth showed an 11-mm-deep pocket with excessive bleeding on probing.

A treatment plan was formulated to include extraction of tooth \#19 with simultaneous guided bone regeneration followed by implant-supported crown replacement, also addressing the infected post-extraction socket and distal root surface of tooth \#20. Local anesthesia was administered by buccal and lingual infiltration of Septanest with adrenaline 1/100,000 (Septanest, Septodont, France) and a short needle. A sulcular incision was made using blade 15c mounted on a \#5A blade handle (Hu-Friedy, Chicago, IL, USA) extending from the mesial aspect of tooth \#20 to the distal aspect of tooth \#18 for surgical accessibility. A pouched mucoperiosteal flap was reflected using a Buser Periosteal Elevator (Hu-Friedy, Chicago, IL, USA), and tooth \#19 was extracted using atraumatic extraction forceps (Hu-Friedy, Chicago, IL, USA) (Fig. 2). The extraction socket was thoroughly debrided to remove all granulation tissues. The distal root surface of tooth \#20 was curetted carefully using a Molt surgical curette (Hu-Friedy, Chicago, IL, USA). Grade II mobility was noted in tooth \#20 due to significant loss of bone support from the distal root aspect. After extraction, the debrided socket showed a two-walled defect with buccal-plate loss, denuded distal root surface of tooth \#20, and damaged inter-radicular bone.

Guided bone regeneration was planned for future implant placement. Three pieces of resorbable premedicated collagen sponge containing lidocaine, chlorhexidine, and metronidazole (Alvanes, Vladmiva, Belgorod, Russia) were adapted to the distal root surface of tooth \#20 to facilitate hemostasis and antimicrobial control of the infected area for proper tissue/bone regeneration of the defect side. A resorbable collagen membrane (T-Barrier Membrane, B\&B Dental Implant Co., Italy) was tacked under a buccal flap to ensure stability upon socket management. An equal-parts mixture of small-sized particulate allograft and xenograft bone materials (ACE Surgical Supply, Brockton, MA) was used to fill the rest of the socket. The collagen membrane was then wrapped around to cover the socket and tacked under the minimally reflected lingual flap. A wound dressing collagen tape (HeliTape; Miltex Integra, Princeton, NJ) was used to cover the collagen membrane and tacked under the buccal- and lingual-flap edges. The site was sutured with mesial and distal simple interrupted knots, and a figure-of-eight technique was used to stabilize the socket contents using 5-0 monofilament polypropylene sutures (SMI sutures, St. Vith, Belgium). The socket seal technique was implemented by application of cyanoacrylate coating (PeriAcryl 90 HV; GluStitch Inc., BC, Canada) on the surgical field (Figs. 3a and 3b). Occlusal adjustment of tooth \#20 was performed to achieve light contact on maximum intercuspation and excursion movements. Postoperative instructions and a prescription of Augmentin $625 \mathrm{mg}$ TID for 7 days, ibuprofen $400 \mathrm{mg}$ prn pain, and Parodontax daily mouthwash for 2 weeks were provided. The patient was recalled 2 weeks later for suture removal and at 1, 4, and 6 months postoperatively for site evaluation. Clinical and radiographic evaluations of the site were performed at the 6-month recall visit to plan for implant surgery (Figs. 4a and 4b).

Following administration of local anesthesia, a flapless and free-hand approach was used to place a $4.2 \times 9$ implant along with the cover screw in a slightly sub-crestal vertical position (Astra Tech Implant EV; Dentsply Sirona, Mannheim, Germany) (Fig. 5). An implant insertion torque of $45 \mathrm{~N} \cdot \mathrm{cm}$ was achieved. The punched-out tissues were adapted and sutured to seal the punched holes (Fig. 6). Postoperative instructions and medication were provided, and the patient was followed up per standard protocol. Three months after 
implant placement, the implant was uncovered following the tissue punch, and a healing abutment of 4$\mathrm{mm}$ height was attached accordingly. Surgical closure and postoperative instructions were provided. An implant-level closed-tray impression technique was performed along with shade-matching 3 weeks after the implant-uncovering procedure. A screw-retained implant-supported zirconia crown was designed, inserted, and torqued to $25 \mathrm{~N} \cdot \mathrm{cm}$ to the internal implant connection (Fig. 7). The screw hole was closed with Teflon tape and a flowable composite. Occlusal adjustment was accomplished with light contact on centric and eccentric movements. The patient was followed up every 3 months in the first year and every 6 months thereafter. The periodontal condition of tooth \#20 improved, and no mobility was noted on recall visits. Post-treatment clinical photographs showed favorable soft-tissue health and level on the buccal and lingual aspects of the implant crown (Figs. 8a and 8b). CBCT radiographs were obtained 1 and 2 years post-treatment and revealed optimum bone quantity and quality around the implant and on the distal root surface of tooth \#20 (Figs. 9 and 10).

The report was conducted in accordance with the Helsinki Declaration of 1975, as revised in 2013. The patient provided written informed consent for the publication of this case report and accompanying figures. Patient anonymity was ensured. The report was exempt from institutional review board approval.

\section{Discussion}

This report presented a clinical approach for application of local pharmaceutical agents together with standard biomaterials for guided tissue and bone regeneration. Although natural teeth may be extracted for many reasons, Osaghae and $\mathrm{Azodo}^{21}$ reported that tooth splitting was the etiological factor for $5 \%$ of the extracted posterior teeth, of which $10 \%$ were mandibular first molars. The prognosis of split teeth with a fracture line extending deep in the sub-gingival area is usually poor $^{22}$; therefore, the teeth in question require replacement with implant or tooth-supported prosthesis.

A meta-analysis ${ }^{23}$ reported no difference in success rates between immediate implant placement in infected and non-infected extraction sockets. However, such treatment options are contraindicated in cases with purulent socket infection and poor bone configuration following extraction. ${ }^{24}$ Accordingly, a more careful approach involving proper implant site preparation prior to placement is feasible to render favorable implant positioning and angulation as described here.

Management of post-extraction sockets is variable. Introduction of hemostatic collagen sponges caused delays in the healing process of the extraction sockets and suppressed differentiation of bone-forming cells. ${ }^{25}$ As a variant of the bone graft form used here, sticky bone resulted in better soft- and hard-tissue healing and regeneration when used for socket grafting following third-molar extraction in a split-mouth clinical study. ${ }^{26}$ Several biological agents have been reported to supplement the standard biomaterials in expediting healing and improving postoperative results following guided tissue and bone-regeneration procedures. ${ }^{27}$ Compared with a collagen-sponge group, supplemental use of recombinant human bone morphogenetic protein 2 with bone graft material resulted in superior bone gain on the distal surfaces of second mandibular molars following third molar extraction. ${ }^{28} \mathrm{~A}$ recently published systematic review and meta-analysis supported the use of platelet-rich fibrin following extraction and reported a faster wound-healing rate and lesser postoperative complications. ${ }^{29}$ The application of readily available agents such as pre-medicated collagen sponges may present a convenient strategic option to supplement readily successful biomaterials for regenerative therapies.

A randomized double-blinded clinical trial ${ }^{30}$ found that repeated application of chlorhexidine-containing chips is beneficial to control sites with active periodontal disease. Local application of controlled-release chlorhexidine chips improved the quantity of bone gain upon tissue-regenerative procedures, and infection control may be crucial for the success of such treatment interventions. ${ }^{19}$ Additionally, evaluation of metronidazole-gel application for tissue regeneration showed that local application of metronidazole gel yielded successful clinical outcomes in treating vertical bony-defect cases along with other biomaterials used for guided tissue regeneration. ${ }^{20}$ However, no study has outlined the effects of combined application of these agents in tissue and bone-regeneration procedures. This report utilized a pre-medicated collagen sponge containing both active agents in a scaffold form, facilitating convenient manipulation and adaptation during the 
surgical procedure and ensuring local release of these agents during the healing process of tissue and bone regeneration.

\section{Conclusion}

Within their limitations, this report suggests that the application of pre-medicated collagen sponges containing chlorhexidine and metronidazole can help control local infection and facilitate significant tissue and bone regeneration in a compromised socket presenting with active and purulent infection, loss of buccal bone plate, and marked vertical bone defects of the adjacent tooth.

\section{References}

1. Yue Yi EK, Siew Ying AL, Mohan M, Menon RK. Prevalence of postoperative infection after tooth extraction: a retrospective study. Int J Dent 2021;2021:6664311.

2. Bortoluzzi MC, Manfro R, De Déa BE, Dutra TC. Incidence of dry socket, alveolar infection, and postoperative pain following the extraction of erupted teeth. J Contemp Dent Pract 2010;11:E033E040.

3. Lindh T, Gunne J, Tillberg A, Molin M. A meta-analysis of implants in partial edentulism. Clin Oral Implants Res 1998;9:80-90.

4. Chen ST, Buser D. Clinical and esthetic outcomes of implants placed in postextraction sites. Int J Oral Maxillofac Implants 2009;24 Suppl:186-217.

5. Ragucci GM, Elnayef B, Criado-Cámara E, Del Amo FS, Hernández-Alfaro F. Immediate implant placement in molar extraction sockets: a systematic review and meta-analysis. Int J Implant Dent 2020;6:40.

6. Lang NP, Pun L, Lau KY, Li KY, Wong MC. A systematic review on survival and success rates of implants placed immediately into fresh extraction sockets after at least 1 year. Clin Oral Implants Res 2012;23 Suppl 5:39-66.

7. Vidal R, Greenwell H, Hill M, Papageorgakopoulos G, Scheetz JP. Success rate of immediate implants placed and restored by novice operators. Implant Dent 2010;19:81-90.

8. Cosyn J, De Lat L, Seyssens L, Doornewaard R, Deschepper E, Vervaeke S. The effectiveness of immediate implant placement for single tooth replacement compared to delayed implant placement: a systematic review and meta-analysis. J Clin Periodontol 2019;46 Suppl 21:224-241.

9. Urban T, Kostopoulos L, Wenzel A. Immediate implant placement in molar regions: risk factors for early failure. Clin Oral Implants Res 2012;23:220-227.

10. Waasdorp JA, Evian CI, Mandracchia M. Immediate placement of implants into infected sites: a systematic review of the literature. J Periodontol 2010;81:801-808.

11. Bell CL, Diehl D, Bell BM, Bell RE. The immediate placement of dental implants into extraction sites with periapical lesions: a retrospective chart review. J Oral Maxillofac Surg 2011;69:1623-1627.

12. Hamouda NI, Mourad SI, El-Kenawy MH, Maria OM. Immediate implant placement into fresh extraction socket in the mandibular molar sites: a preliminary study of a modified insertion technique. Clin Implant Dent Relat Res 2015;17 Suppl 1:e107-e116.

13. Kfir E, Kfir V, Kaluski E. Immediate bone augmentation after infected tooth extraction using titanium membranes. J Oral Implantol 2007;33:133-138.

14. Zucchelli G, Sforza NM, Clauser C, Cesari C, De Sanctis M. Topical and systemic antimicrobial therapy in guided tissue regeneration. J Periodontol 1999;70:239-247.

15. Minabe M, Kodama T, Kogou T, et al. Clinical significance of antibiotic therapy in guided tissue regeneration with a resorbable membrane. Periodontal Clin Investig 2001;23:20-30.

16. Payer M, Tan WC, Han J, et al. The effect of systemic antibiotics on clinical and patient-reported outcome measures of oral implant therapy with simultaneous guided bone regeneration. Clin Oral Implants Res 2020;31:442-451.

17. Pietruska M, Dolińska E, Milewski R, Sculean A. Effect of systemic antibiotics on the outcomes of regenerative periodontal surgery in intrabony defects: a randomized, controlled, clinical study. Clin Oral Investig 2021;25:2959-2968. 
18. Abu-Ta'a M. Adjunctive systemic antimicrobial therapy vs asepsis in conjunction with guided tissue regeneration: a randomized, controlled clinical trial. J Contemp Dent Pract 2016;17:3-6.

19. Reddy MS, Jeffcoat MK, Geurs NC, et al. Efficacy of controlled-release subgingival chlorhexidine to enhance periodontal regeneration. J Periodontol 2003;74:411-419.

20. Sander L, Frandsen EV, Arnbjerg D, Warrer K, Karring T. Effect of local metronidazole application on periodontal healing following guided tissue regeneration. Clinical findings. J Periodontol 1994;65:914920.

21. Osaghae IP, Azodo CC. Analysis of split tooth as an unstudied reason for tooth extraction. BMC Res Notes 2014;7:630.

22. Endodontics: Colleagues for Excellence Newsletter, Fall/Winter; 1997. American Association of Endodontics. Cracking the Cracked Tooth Code.

23. Lee J, Park D, Koo KT, Seol YJ, Lee YM. Comparison of immediate implant placement in infected and non-infected extraction sockets: a systematic review and meta-analysis. Acta Odontol Scand 2018;76:338-345.

24. Ebenezer V, Balakrishnan K, Asir RV, Sragunar B. Immediate placement of endosseous implants into the extraction sockets. J Pharm Bioallied Sci 2015;7 Suppl 1:S234-S237.

25. Guo X, Hu H, Liu Y, Bao C, Wang L. The effect of haemostatic agents on early healing of the extraction socket. J Clin Periodontol 2019;46:766-775.

26. Rupawala TA, Patel SM, Shah NH, Sanghvi KB, Makwana SV, Bhimani KK. Efficacy of sticky bone as a novel autologous graft for mandibular third molar extraction socket healing - an evaluative study. Ann Maxillofac Surg 2020;10:335-343.

27. Bashutski JD, Wang HL. Biologic agents to promote periodontal regeneration and bone augmentation. Clin Adv Periodontics 2011;1:80-87.

28. Ku JK, Jeong YK. Effectiveness of bone graft for an alveolar defect on adjacent second molar after impacted mandibular third molar extraction. J Oral Maxillofac Surg 2021;79:756-762.

29. Fujioka-Kobayashi M, Miron RJ, Moraschini V, Zhang Y, Gruber R, Wang HL. Efficacy of platelet-rich fibrin on socket healing after mandibular third molar extractions. J Oral Maxillofac Surg Med Pathol 2021;33:379-388.

30. Machtei EE, Hirsh I, Falah M, Shoshani E, Avramoff A, Penhasi A. Multiple applications of flurbiprofen and chlorhexidine chips in patients with chronic periodontitis: a randomized, double blind, parallel, 2-arms clinical trial. J Clin Periodontol 2011;38:1037-1043.

\section{Figure Legends}

Figure 1. Pre-treatment radiograph indicating split tooth \#19 and significant infection around its mesial root and distal root surface of tooth \#20.

Figure 2 . Extracted tooth \#19 showing extension of the fracture line and the size of the radicular granuloma.

Figure 3 . A composite image illustrating (a) surgical closure using the socket seal technique after extraction of tooth \#19 and (b) socket fill following extraction with radiolucent gap on the distal root surface of tooth $\# 20$, which represents pre-medicated collagen sponges.

Figure 4. A composite figure illustrating (a) clinical view of site \#19 6 months following extraction and GBR procedures and (b) radiographic evaluation of site \#19 showing progressive healing and bone fill.

Figure 5 . Postoperative view of the implant following placement in a two-stage surgical approach.

Figure 6 . Postoperative clinical view of the implant site following closure of the surgical hole using the excised native soft tissue.

Figure 7 . Postoperative clinical view of the screw-retained implant crown following insertion.

Figure 8. A composite figure illustrating clinical views of the implant-supported crown from the (a) buccal and (b) lingual aspects 2 years after treatment completion. 
Figure 9 . CBCT radiograph obtained the year following completion of treatment, indicating progressive bone gain around the implant and the distal root surface of tooth \#20.

Figure 10. CBCT radiograph obtained 2 years following completion of treatment indicating adequate bone fill and maturation around the implant and distal root surface of tooth \#20.

\section{Hosted file}

Title page.docx available at https://authorea.com/users/339304/articles/558711-applicationof-pre-medicated-collagen-sponges-with-regenerative-biomaterials-for-management-of-aninfected-extraction-socket

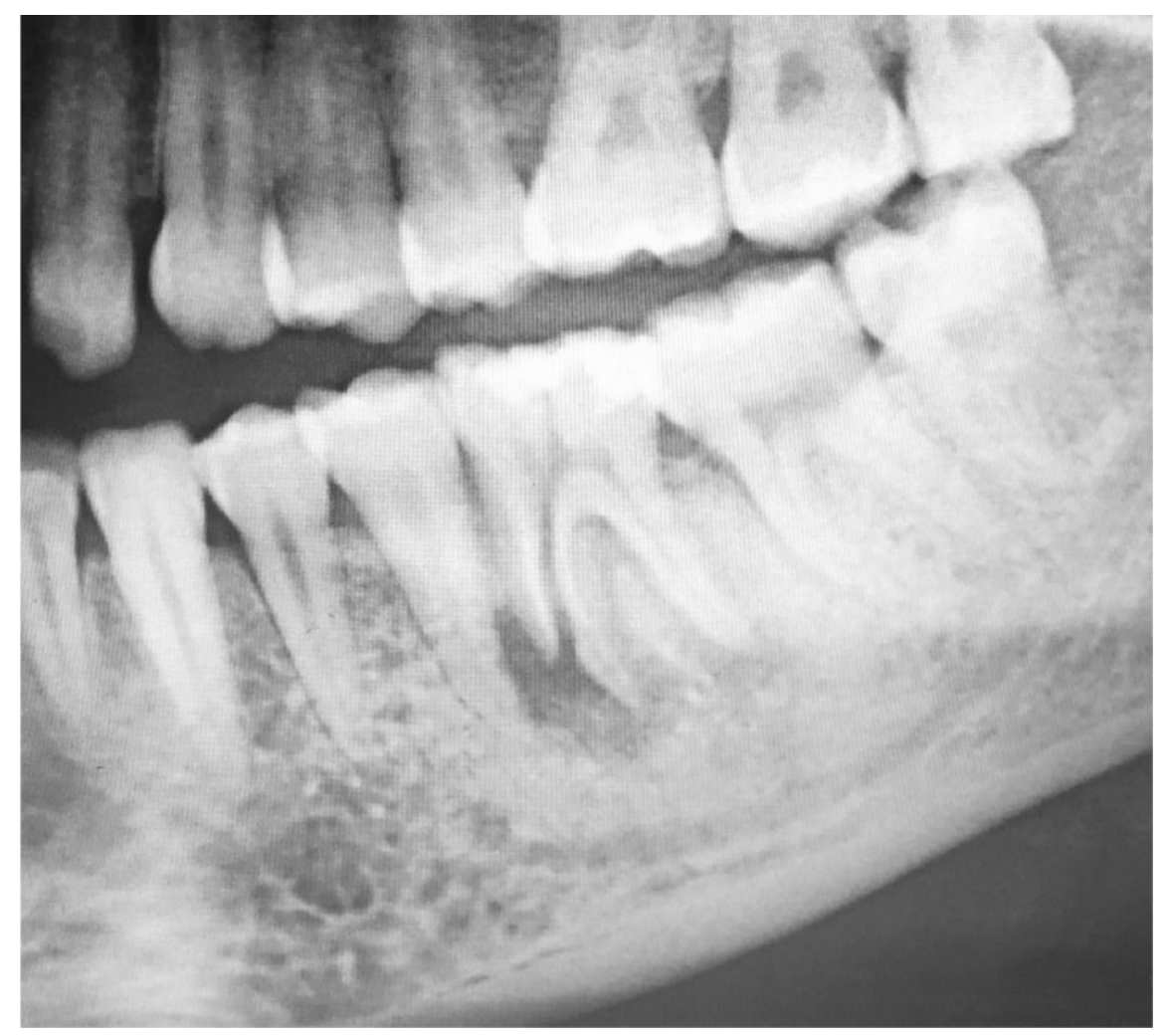



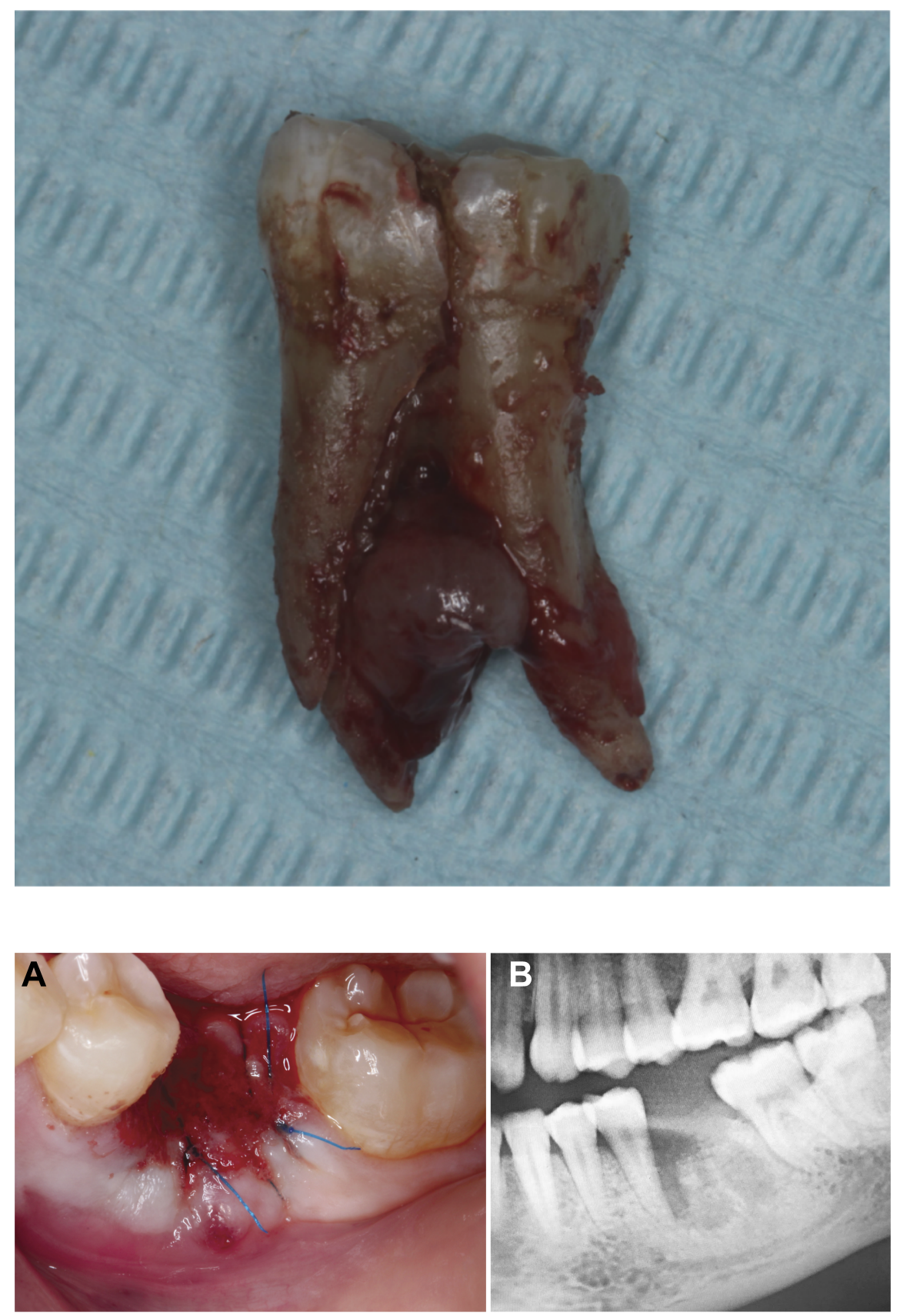

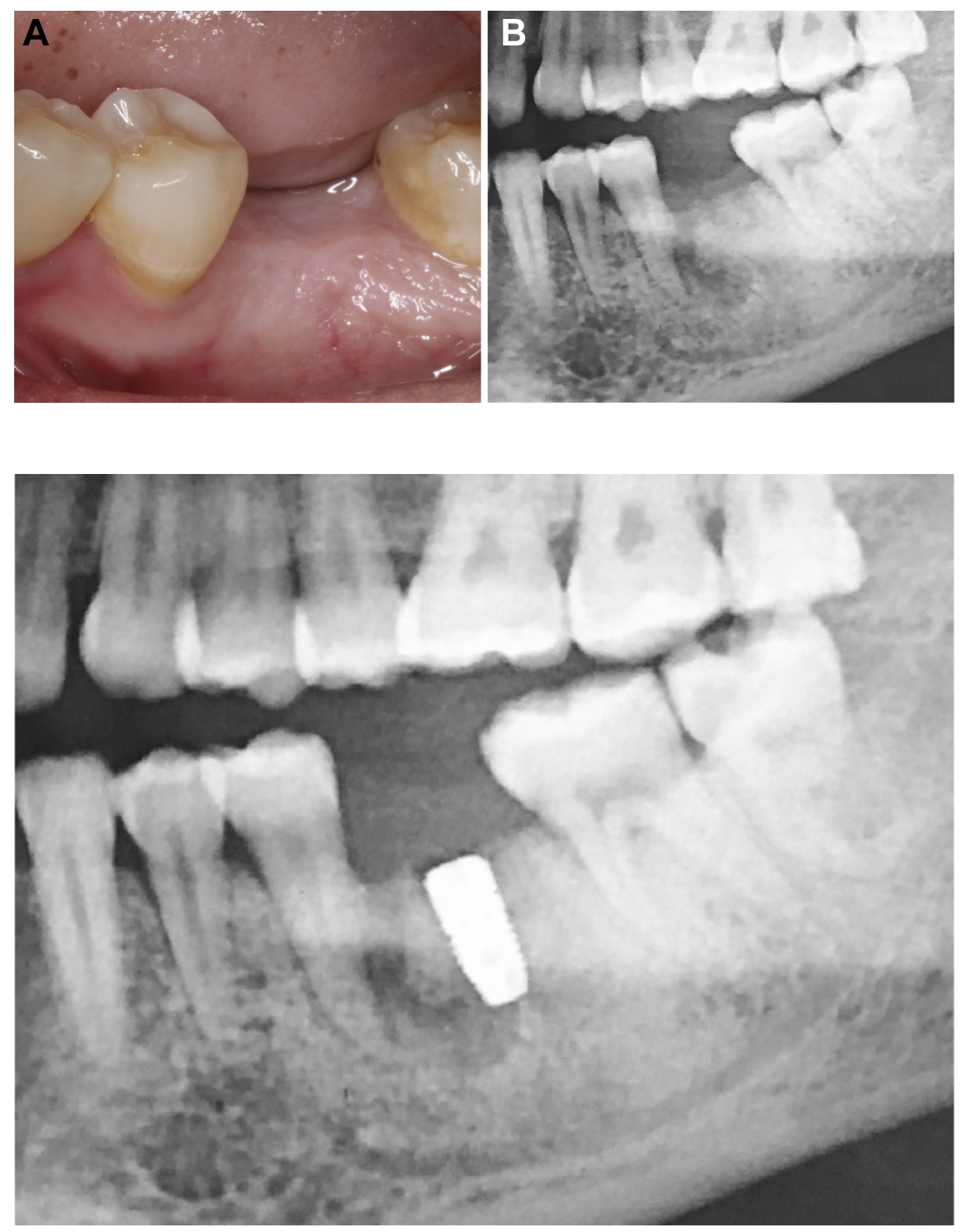

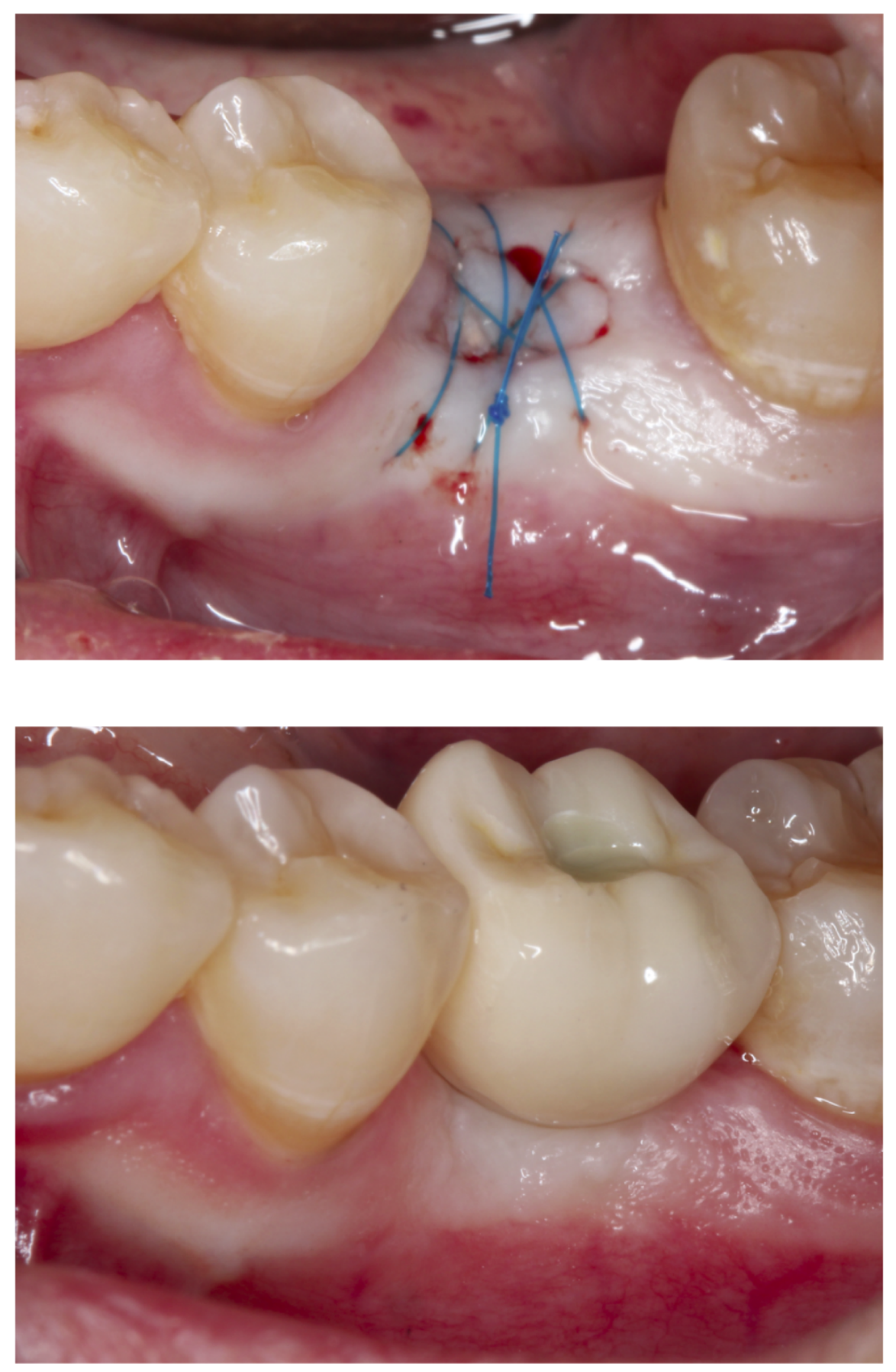

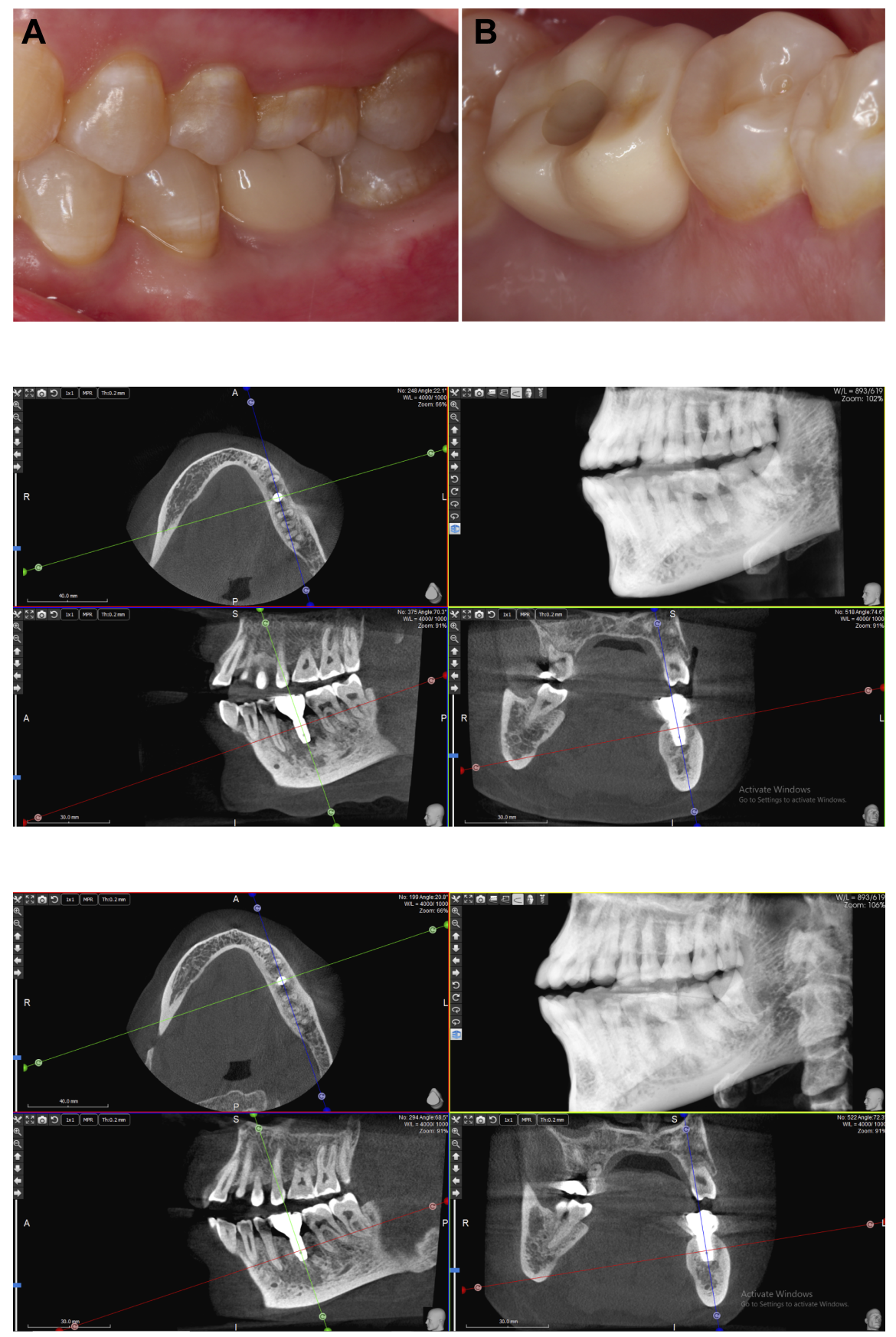